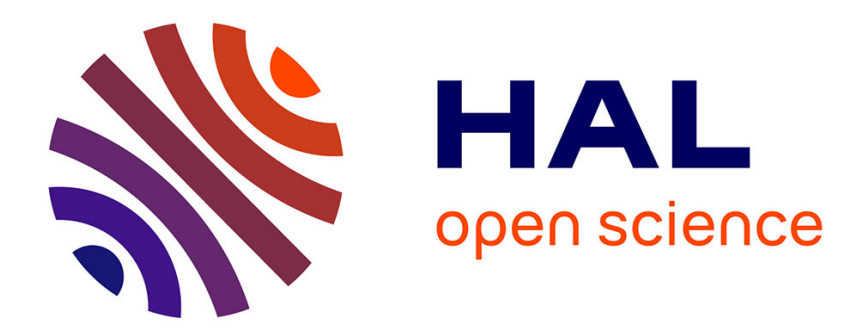

\title{
La réforme des diplômes en travail social, du côté des centres de formation
}

Silvia Valentim

\section{To cite this version:}

Silvia Valentim. La réforme des diplômes en travail social, du côté des centres de formation. Les Métiers de la petite enfance, 2019, 25, pp.19 - 21. 10.1016/j.melaen.2019.06.006 . hal-03487978

\section{HAL Id: hal-03487978 \\ https://hal.science/hal-03487978}

Submitted on 20 Dec 2021

HAL is a multi-disciplinary open access archive for the deposit and dissemination of scientific research documents, whether they are published or not. The documents may come from teaching and research institutions in France or abroad, or from public or private research centers.
L'archive ouverte pluridisciplinaire HAL, est destinée au dépôt et à la diffusion de documents scientifiques de niveau recherche, publiés ou non, émanant des établissements d'enseignement et de recherche français ou étrangers, des laboratoires publics ou privés.

\section{다)(1) $(5$}

Distributed under a Creative Commons Attribution - NonCommerciall 4.0 International 


\section{Dochead dossier}

\section{Sous-dochead La refonte des diplômes de niveau III en travail social analyse}

\section{La réforme des diplômes en travail social, vue du côté des centres de formation}

\section{Silvia Valentim}

Chercheure-associée au laboratoire EMA (École, Mutation, Apprentissage)

Laboratoire EMA, université de Cergy-Pontoise, site de Gennevilliers, ZAC des Barbanniers, avenue Marcel-Paul, 92230 Gennevilliers, France

Adresse e-mail : silvia.valentim@gmail.com (S. Valentim).

\section{Résumé}

La réforme des formations en travail social est en vigueur depuis septembre 2018. Elle a eu des conséquences importantes pour les centres de formation, notamment concernant le processus d'accréditation par les universités. Analyse.

(C) 2019

Mots clés - accréditation; centre de formation; formation professionnelle ; institution ; réforme ; travail social

Chaque centre de formation, comme toute institution rendant un service public, entretient un territoire, l'occupe et est ancré dans l'histoire de celui-ci. La réforme des formations en travail social, en vigueur dans le secteur depuis le $1^{\text {er }}$ septembre 2018 [1], n'est pas sans conséquences pour les centres de formation et les logiques territoriales.

\section{T1 Une mise en œuvre mouvementée}

Du côté des centres de formation, la réforme est vécue de manière différente en fonction des caractéristiques de chaque établissement. Il est encore difficile d'évoquer ses effets au vu de 
la courte période de mise en œuvre. Néanmoins, nous pouvons nous interroger sur les conditions d'émergence de cette réforme et rappeler qu'elle s'inscrit dans un ensemble de changements majeurs pour le secteur.

Le travail social vit un moment fort de son institutionnalisation [2]. Il intègre par le biais du processus d'accréditation le dispositif universitaire, et le niveau de la formation est reconnu et gradué selon les principes de l'université.

Pour cela, depuis 2018, les centres de formation et les universités cherchent à rapprocher leurs logiques de fonctionnement et leurs contraintes. Ce rapprochement est loin d'être sans conséquences pour les personnes qui font vivre ces établissements. En analyse institutionnelle, les changements répétés font référence au processus d'institutionnalisation constant, que nous observons dans différents secteurs de la société depuis quelques années : nous avons à peine le temps de nous habituer à une organisation, que celle-ci est à nouveau en mouvement et en changement.

La réforme des diplômes en travail social a pour caractéristique de s'accompagner de différents mouvements, tous importants et comportant des enjeux non négligeables. En effet, la nouvelle gouvernance en termes de formation professionnelle, la reforme de celle-ci, viennent complexifier la mise en œuvre des formations. "La réglementation prévoit un double régime d'ouverture des formations : d'une part, l'agrément, relevant de la compétence $d u$ président du conseil régional et, d'autre part, l'autorisation d'ouverture accordée par le ministre chargé de l'enseignement supérieur. Les deux procédures sont indépendantes, mais doivent s'articuler. »[3] Ainsi, la concertation entre les trois pouvoirs : conseil régional, direction régionale de la jeunesse, des sports et de la cohésion sociale (DRJSCS) préfet, direction générale de l'enseignement supérieur et de l'insertion professionnelle du ministère de l'Enseignement supérieur, de la Recherche et de l'Innovation (DGESIP), devient une priorité pour la bonne marche de la réforme. Ce processus est différent en fonction des régions.

\section{T1 Des accréditations, des disparités marquantes}

La mise en œuvre des processus d'accréditation ne se déroule pas de manière uniforme sur l'ensemble du territoire français, ni même au sein d'une même région. La connaissance du secteur du travail social par les universités (et rectorats) semble être déterminante pour la mise en place du processus. Par exemple, tout en étant piloté par le rectorat et la DRJSCS dans la 
région Hauts-de-France, le processus a eu des difficultés à déboucher sur un accord, alors que d'autres conventions existent avec l'université de Lille autour de la licence sciences de l'éducation. Ici le manque de connaissance du processus et de ses effets à long terme pour l'université n'était pas suffisamment maîtrisé pour permettre qu'un accord soit trouvé.

TEG1 De manière générale, l'université ne semble pas disposer des moyens humains nécessaires pour répondre aux exigences de la réforme :

- disponibilités des enseignants-chercheurs pour des cours dont la volumétrie n'est pas déterminée ;

- présence lors des commissions pédagogiques et de perfectionnement ;

- et, de manière optionnelle, participation aux jurys des épreuves de certification (sauf pour le mémoire des pratiques professionnelles : présence obligatoire).

TEG1 Par ailleurs, les conséquences incluent des questions de légitimité. En effet, les centres de formation en travail social se caractérisent par le fait d'être composés principalement de formateurs issus du monde professionnel, ayant obtenu des diplômes d'État, en dehors des logiques universitaires. Cette situation réveille des ressentis liés au rapport entre université et travail social. La première, par le savoir qui lui est reconnu, son influence et la validation des pratiques du second. Cette réalité est d'autant plus saillante que les textes ne valorisent pas les ressources humaines des centres de formations, car il existe des formateurs en travail social en possession de masters, inscrits dans un processus doctoral, ayant un doctorat et qualifiés dans leurs disciplines par le Conseil national des universités. Ces formateurs en travail social interviennent au sein des licences et des masters en assurant des cours magistraux et des travaux dirigés, comblant ainsi le manque d'enseignant-chercheur à l'université.

TEG1 Notons aussi que le décret du 22 août 2018 ne contient pas de définition de l'enseignant-chercheur. Toutefois, celui-ci semble être décrit, par les DRJSCS et les rectorats, comme une personne ayant le statut de maître de conférences et ayant un poste à l'université. Ce titre, ainsi que la recherche, seraient-ils un monopole de l'université ? Au-delà des aspects statutaires, quel est le message de l'État quant aux recherches menées dans ce secteur, en dehors de l'université ? Comment mettre en œuvre des textes qui semblent manquer de cohérence?

TEG1 Enfin, nous pouvons nous questionner sur les différences entre accréditation et universitarisation, notamment en comparaison avec ce que nous avons pu observer quant à la 
formation des infirmières. "L'année 2009 voit naître les Groupements de Coopération Sanitaire, qui fixent la convention tripartite entre la Région qui finance [la] formation [des infirmières], les universités et les IFSI [Instituts de formation en soins infirmiers], mais également le grade licence. » [4] Certes, il ne s'agit pas des mêmes contenus et réalités, mais savons-nous vraiment ce que marquent les différences? Nous semblons loin de la transparence des informations et des enjeux concernant les visées de la réforme.

L'un des effets de l'institutionnalisation constante est de créer une "opacité", par le trop grand nombre d'informations transmises et la non-maîtrise des enjeux, qui rend le travail de compréhension de l'institution difficile à ceux qui la font vivre dans le quotidien des centres de formation.

\section{T1 Une réforme pourtant attendue}

Concernant la formation des éducateurs de jeunes enfants (EJE) notamment, la réforme était attendue pour faire évoluer le cadre réglementaire en lien avec la réalité d'un terrain professionnel très mouvementée. Les textes font état des évolutions, la mise à jour de certains contenus en est une réalité.

Par ailleurs, il n'existe plus de formation de niveau IV ni III dans le secteur de la petite enfance. Cela risque de creuser l'écart entre les professionnels sur le terrain, ce qui aura certainement des conséquences sur le travail quotidien dans les structures, notamment en termes de nombre et de niveau de formation des professionnels présents auprès des enfants.

\section{T1 Quel avenir pour les diplômes d'État ?}

La vitesse avec laquelle les informations parviennent aux établissements et les échéances auxquelles elles doivent être traitées vont sans doute marquer les différentes réalités du secteur du travail social. Entre les grands centres de formation, disposant des moyens humains et matériels plus importants, et les plus petits établissements en termes d'effectifs et d'offres de formation, l'écart risque de se creuser, d'autant plus que les nouvelles modalités de certifications, non accompagnées des moyens supplémentaires pour les mettre en œuvre, questionnent sur la faisabilité. 
En effet, les mois qui ont précédé la publication des textes officiels se sont accompagnés de nombreuses interrogations dans les services. À tous les niveaux, administratifs et pédagogiques, chacun savait que les changements à venir pouvaient provoquer des effets plus ou moins maîtrisables, à court et long termes.

Le changement majeur en ce sens est l'intégration du dispositif d'admission Parcoursup. Il s'agit non seulement d'un nouvel outil à maîtriser, mais aussi d'un changement de culture pour les écoles de formation en travail social, habituées à organiser un processus de sélection de ses futurs étudiants. À cela s'ajoute le fait que Parcoursup est un dispositif récent, mis en place seulement depuis un an.

Le processus d'admission est indispensable pour la reconnaissance des écoles. En général, les candidats classent les établissements par la sélection à l'entrée et le taux de réussite aux examens. Dans les années à venir, il sera important d'accompagner les processus d'entrée et de sortie en formation, afin de mesurer les effets de ces changements, avant d'en mettre en place des nouveaux...

\section{T1 Les certifications}

À l'étude des différents textes [3], pas de surprise majeure, les contenus semblent déplacés entre les domaines de formation (DF), toutefois le cœur des métiers ne subit pas de transformations profondes.

Les changements conséquents concernent des modalités de certification. Les centres de formation agréés par les régions et accrédités par l'université deviennent des certificateurs sous le contrôle de la DRJSCS. Cette dernière garde la responsabilité de l'épreuve de certification du mémoire de pratique professionnelle. Deux épreuves orales sont ajoutées aux DF communs aux cinq diplômes du travail social : DF3 et DF4. Ces oraux complètent des travaux écrits remis par les étudiants.

Mais comment mettre en place cette nouvelle délégation, sans soutien financier de l'État?

\section{T1 Une solidarité à l'œuvre}


Entre les acteurs de la formation, que ce soit dans les régions ou au niveau national, une certaine forme de solidarité continue à exister et semble s'être renforcée autour du partage de lectures et de la compréhension des nouveaux textes.

Par exemple, l'Association pour la formation au métier d'EJE (Aformeje) [5] constitue un espace d'échanges indispensable pour la mise en place de la réforme. Les réunions régulières permettent de suivre l'avancée des travaux et d'échanger sur les nombreux questionnements qu'ils suscitent.

\section{T1 Conclusion}

La manière dont se déroule le processus de cette réforme questionne quant à la compréhension des enjeux de la formation initiale en travail social. Les textes, remis fin août 2018 pour une mise en œuvre dès le mois de septembre, semblent non terminés et manquent de précisions sur des points primordiaux pour la validation du diplôme d'État.

L'année 2019 ne peut être qu'une ébauche de ce que seront les années à venir, lorsque chacun à son niveau se sera approprié des nouveaux textes. La prudence est donc de mise.

Une telle réforme, événement institutionnel majeur pour un secteur, donne à voir les limites de l'institution à instaurer un véritable dialogue et à coordonner le travail de réflexion autour des diplômes. Malgré tout, l'institution fera vivre la réforme et construira du sens. Reste à espérer que ce sens émergera de la reconnaissance du travail [6] qui sera réalisé.

\section{Déclaration de liens d'intérêts}

L'auteur déclare ne pas avoir de liens d'intérêts.

\section{Références}

[1] Décret n ${ }^{0}$ 2018-734 du 22 août 2018 relatif aux formations et diplômes du travail social. www.legifrance.gouv.fr/affichTexte.do?cidTexte=JORFTEXT000037324454\&categorieLien $=\mathrm{id}$

[2] Lourau R. (1970). L’analyse institutionnelle. Paris: Les Éditions de Minuit; 1970. 
[3] Ministère des Solidarités et de la Santé. Ministère de l'Enseignement supérieur de la Recherche et de l'Innovation. Fiches techniques à destination des acteurs de la mise en œuvre de la réingénierie des formations des 5 diplômes du travail social de niveau II. 2 octobre 2018.

[4] Effantin J, Jourdan A, Peguet-Revuz J. Historique de la formation en soins infirmiers. 17 mai 2018 sur le site http://fnesi.org/2018/05/historique-de-la-formation-en-soins-infirmiers/

[5] Association pour la formation au métier d'éducateurs de jeunes enfants. https://frfr.facebook.com/pages/category/Organization/Aformeje-953818574633126/

[6] Fender R, Mangematin Y, Husson J, et al. Redonner du sens au travail : essai de modélisation de la reconnaissance au travail. Projectics / Proyéctica / Projectique. 8-9(2):2011;51-65.

\section{Illustration}

\section{Valentim-Illus1}

(C) /stock.adobe.com

Légende: Depuis 2018, les centres de formation et les universités cherchent à rapprocher leurs logiques de fonctionnement et leurs contraintes. 


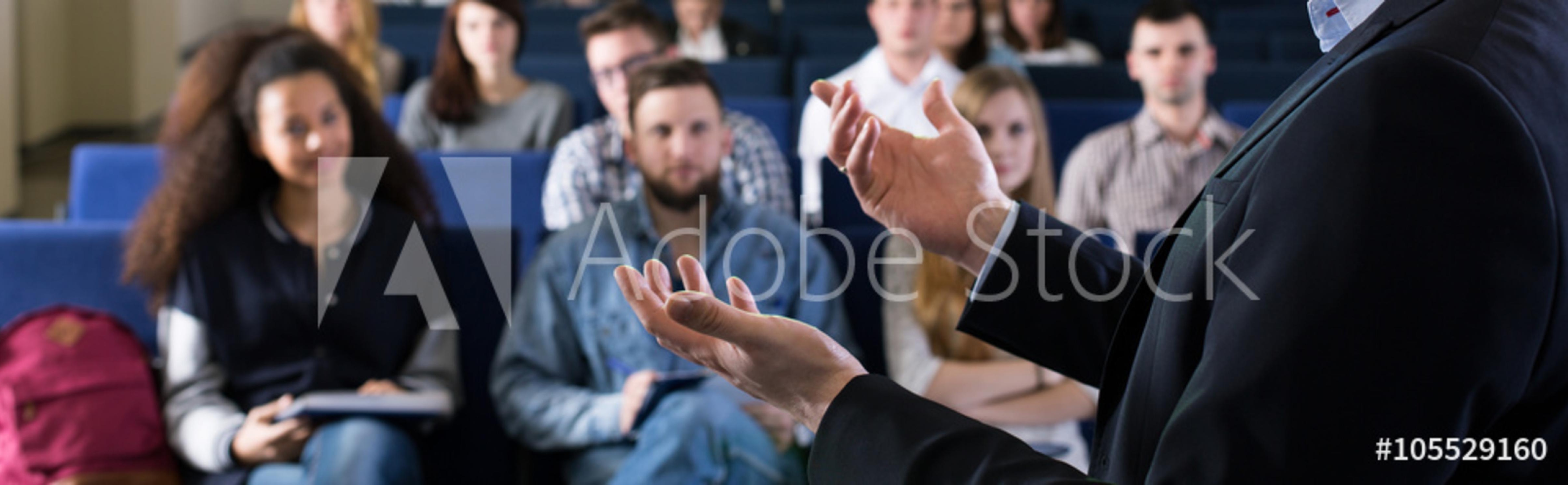

